Historic, Archive Document

Do not assume content reflects current scientific knowledge, policies, or practices. 



\section{Special Fall Sale}

of Choice

Gladiolus Bulbs

Bargain Prices

for November

and December

Delivery Only

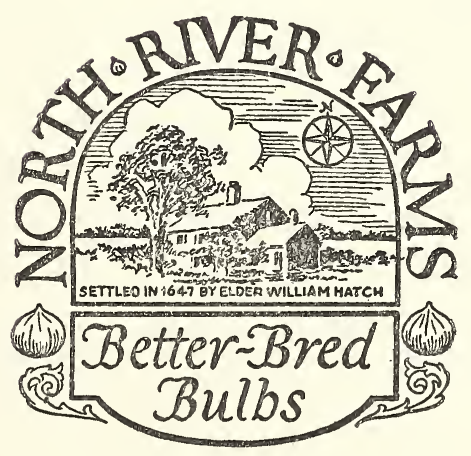

NORTH RIVER FARMS

Marshfield, Mass. 
We have a large part of our stock of Gladiolus bulbs already dug, and we are going to have many more than we wish to store over winter. Therefore we are again offering a large number of varieties at such low prices that it will pay you to store the bulbs until planting time. Our bulbs are clean and plump.

AdMiRATION, Lavender pink

ADORA, Creamy white

Alice Tiplady, Otange salmon

$\$ .255$

. I 12

ARden, Red (Prim)

ATREUS, Rose pink

$.05 \quad 25$

. I O 13

.158

.158

. I O I 2

. I 58

$.08 \quad 15$

.503

. I O I 2

. I 2 I 0

$.60 \quad 2$

$.08 \quad 15$

. $10 \quad 12$

. I O I 2

. I 58

.158

$.08 \quad 15$

. I O I 2

$\begin{array}{llr}\text { DIENER'S AMERICAN BEAUTY, Rose } & .20 & 6 \\ \text { DOROTHY WHEELER, Pale rose pink } & .05 & 25\end{array}$

DORRITT, Light pink .I 5

DR. W. VAN FleET, Tall early pink . I 58

DUCHESS OF YORK, Royal putple $\quad .20 \quad 6$

EDA, Dark orange salmon . I 12

E. J. SHAYLOR, Rose pink $\quad .08$ I5

ELEGANT BEAUTY, Grenadine pink . I 58

ELF, Yellow bud, white flower . I 12

Elizabeth Hardee, Rose flaked ted .60 2

ElizABETH TABOR, Pink, blotched . I 58

ELORA, Cream white, yellow throat . I 12

ELSE Rose, Pink $\quad .206$

ESTHER OLSEN, Sea-shell pink $\quad .502$

ETENDARD,Cteam .I 58

EVELYN KIRTLAND, Rosy pink $\quad .08$ I 5

Fair Columbian, Lilac-pink $\quad .20 \quad 6$

Fairland, Vermilion-scarlet . Io I 2

FaIRY Blossom, White, yellow throat .20 6

F. J. SYMMES, Rose pink . I 12

FLORA, Yellow $\quad .08$ I 5

GEN. KUROKI, Rosy red $\quad .50$

GiAnt NyMPH, Tall light pink .I5 8

GLOWING, Datk-ted 2.50

GOLD DROP, Marguerite-yellow $\quad$. I5 8

GOLDEN GIRL, Yellow prim $\quad .05 \quad 25$

Golden Glow, Yellow $\quad .255$

GREGOR MENDEL, Wine purple $\quad .25 \quad 5$

HERADA, Mauve .08 I 5

HESTIA, Otange-salmon .I 0 I 2

$\begin{array}{lll}\text { IDA VAN, Scarlet-red } & .05 \quad 25\end{array}$

IMMENSITY, Salmon $\quad .25 \quad 5$ 
Storing and caring for Gladiolus bulbs over winter is as easy as storing and keeping potatoes. Simply keep them in a cool, dry place, being sure that the temperature never goes below freezing. That is all there is to it, and when planting time comes your bulbs are ready, and you avoid the Spring rush which sometimes causes annoyance.

INDIAN MAID, Peach-blossom pink \$ Each $\underset{\$ 1.00}{\text { For }}$ JEWELL, Pink with yellow throat JoE Coleman, Rich red, ruffled .08 I 5 JUNO, Orange-scarlet .I 12 KIRCHOFF's VIOLET, Best violet 1.00 I KIVA, Salmon-pink $\quad$ r.00 I LiLAC UNIQUE, Clear lilac $\quad .255$ LILITH, Mottled pink $\quad .20 \quad 6$ Lillian HARris Coffin, Rose-pink .60 LOUISE, Lavender $\quad$. I5 8 LUSTRE, Bright-red . I 58 MADISON COOPER, Satiny-ted $\quad .206$ MAINE, White .I 58 MAJESTIC, Red $\quad$. $10 \quad 12$ MARIE KUNDERD, Ruffled white $.25 \quad 5$ MARIETTA, Geranium pink $\quad .503$ MATTHEW CRAWFORD, Ivory $\quad .20 \quad 6$ MISHAWAKa, Yellow Miss Helen Franklin, White $\quad 0525$ MIsS SPOKANE, Salmon-orange .I 58 MMe. Mounnet-Sully, Milk-white .20 6 MORNING GLORY, Yellow $\quad$.I 58 Mr. H. A. HydE, Snow-white .255 MrS. Dr. NORTON, Shell-pink .0525 MrS. F. C. Peters, Lavendet $\quad .206$ MrS. JoHn S. WoOD, Pink $\quad .403$ MRS. L. S. SCHWEPPE, Tyrian-pink .40 3 MrS. Rudolph SPRECKLeS, Pink .io i 2 MYRA, Large pink prim $\quad .08$ I 5 Myrtle, Rose-pink $\quad .08$ I 5 NYDIA, Salmon .I 59

ORANGE Glory, Peach-red $\quad .08$ I 5 ORIENTA, Crushed strawberty $\quad .20 \quad 6$ ORPHEUM, Rose $\quad .25 \quad 5$ PANTHEON, White .I5 8

PARMA, Rose-pink and lilac $\quad .25 \quad 5$

PINK WONDER, Pink . I5 8

Pola Negri, Yellow and apricot .20 6

PRIDE OF HILlEGOM, Bright-red . Io I 2

PRIDE OF LANCASTER, Orange . Io I 2

Primrose Beauty, Yellow

PRIMULINUS HYBRIDS, 30

Primunella, Buff yellow $\quad$ in 8

PRINCE OF WALES, Salmon $\quad .05 \quad 25$

PRINCELY, White 1.00 I

Priority, Pink $\quad .503$

Proserpine, Btight rose-ted $\quad .08$ I 5

PythiA, Giant red . I 58

$\begin{array}{lll}\text { RARITY, Lavender } & .20 & 6\end{array}$

RED COPPER, Salmon-tose $\quad$. I 58

REMEMBRANCE, Rose-pink I . I $\quad$ I. 3 
RitA, Yellow

ROMANCE, Orange red

RosAliND, Datk rose-ted

ROSE AsH, Ashes of toses

ROSE UNIQUE, Rose

ROSY GLOW, Rose pink on white

RUfFled AMERICA, Ruffled pink

SALMON BEAUTY, Salmon pink

Salmon Plume, Flesh

SAlOME JANE, Salmon

SCARLET PRINCEPS, Bright red

SCARLET WONDER, Scarlet

SEABROOK GEM, Lavender

SEBESTA, White, yellow throat

SERRATA, Straw-coloted

SHARON ROSE, Rose-pink

SHEILA, Light salmon

SHELl PINK, Rose-pink

SidNey PlumMer, Yellow

SNAPDRAGON, Red and yellow

SNOW GLORY, Ruffled white

SweETHEART, Cream white

SWEET LAVENDER, Lavender pink

SUNNYMEDE, Orange

TAWNY, Tan

THISTLE, Rose red

THOS. T. KENT, Hermosa-pink

TWILIGHT, Ivory flaked pink

TWINKLES, Salmon-buff

UHLAN CHIEF, Vermilion-scarlet

VANITY, Light pink

VIOLET GLORY, Violet

WILLIS E. FRYER, Mallow-putple

YELLOW GLORY, Yellow

ZENOBIA, Otange prim

SELECT MIXTURE,

StANDARd MixtURE,

This sale ends and all prices are withdrawn December I $5,1926$.

These prices are for bulbs I I/4" up, and include free delivery. If desired, two half-sized bulbs will be furnished for the price of one large bulb.

We guarantee our stock to be true to name, but only to the extent that we will replace, free of charge, any bulbs found to be otherwise.

All stock offered subject to prior sale. If we are sold out of large bulbs, we reserve the right to substitute a larger number of smaller bulbs, or, in the case of small quantities, another variety of equal or greater value, unless instructed not to do so.

Our illustrated annual catalog will be sent you about January I. It will not contain many of the varieties listed here.

Our wholesale price list is now ready, and will be sent on application if you desire to purchase larger quantities. 